\title{
Aggregation Forms of Type I Collagen Studied by Simultaneous Small- and Wide-Angle X-ray Scattering
}

\section{Ying-Jen Shiu, ${ }^{1}$ Kuei-Fen Liao, ${ }^{1}$ Je-Wei Chang, ${ }^{1}$ Orion Shih, ${ }^{1}$ Chen-An Wang, ${ }^{1}$ Ting-Wei Hsu, ${ }^{1}$ Tzu-Jung Lan, ${ }^{1}$ Yung-Chen Lin, ${ }^{1}$ Chia-Yu Lin, ${ }^{1}$ and U-Ser Jeng ${ }^{1,2^{*}}$}

\author{
${ }^{I}$ National Synchrotron Radiation Research Center, Hsinchu Science Park, Hsinchu 30076, Taiwan; \\ ${ }^{2}$ Department of Chemical Engineering, National Tsing Hua University, Hsinchu 30013, Taiwan;
}

\author{
usjeng@nsrrc.org.tw
}

Type I collagen solution (bovine skin based) is studied using the biological small- and wide-angle X-ray scattering beamline at the 3.0 GeV Taiwan Photon Source of the National Synchrotron Radiation Research Center. Concomitant SAXS-WAXS data are collected from the sample elution with an online size exclusion column (SEC) of HPLC, incorporated with UV-vis absorption followed by refractive of index measurements. SEC-SAXS result indicates a relatively monodisperse size distribution of the tropocollagen, which comprises three left-handed helices of polypeptide strands that are twisted together into a right-handed coiled coil for a triple helix. The SAXS-revealed gyration $\mathrm{R}_{\mathrm{g}}$ of $195 \AA$ and elongated shape together with the molecular mass and the hydrodynamic radius $\mathrm{R}_{\mathrm{h}}$ measured from dynamic light scattering and multi-angle laser light scattering, together, indicate a dimer form of the tropocollagen. Interestingly, these dimers can gradually form visible networks in solution upon adding short peptides; further, circular dichroism result indicates that these peptides are fond to reserve better the secondary structure of tropocollagen in solution upon UV illumination. The network formation mechanism of tropocollagen will be discussed in terms of the interaction of tropocollagen with the short peptides.

Keywords: tropocollagen, peptides, SAXS, WAXS. 